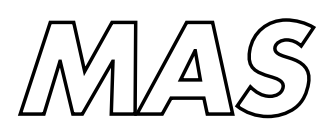

Modelling, Analysis and Simulation

Modelling, Analysis and Simulation
MAS Kinetic electrostatic structures in current-carrying pair
plasmas

A. Luque, H. Schamel, B. Eliasson, P.K. Shukla

Report MAS-R0606 February 2006 
Centrum voor Wiskunde en Informatica (CWI) is the national research institute for Mathematics and Computer Science. It is sponsored by the Netherlands Organisation for Scientific Research (NWO).

CWI is a founding member of ERCIM, the European Research Consortium for Informatics and Mathematics.

CWI's research has a theme-oriented structure and is grouped into four clusters. Listed below are the names of the clusters and in parentheses their acronyms.

Probability, Networks and Algorithms (PNA)

Software Engineering (SEN)

\section{Modelling, Analysis and Simulation (MAS)}

Information Systems (INS)

Copyright (C) 2006, Stichting Centrum voor Wiskunde en Informatica

P.O. Box 94079, 1090 GB Amsterdam (NL)

Kruislaan 413, 1098 SJ Amsterdam (NL)

Telephone +31205929333

Telefax +31205924199

ISSN 1386-3703 


\title{
Kinetic electrostatic structures in current-carrying pair plasmas
}

\begin{abstract}
The existence and properties of phase-space structures in current-carrying pair plasmas is studied by means of the finite amplitude expressions of the pseudo-potential method. Emphasis is given to double layers, solitary structures and periodic waves. The results can be applied to electron-positron plasmas as well as to plasmas containing heavier charged particles with the same mass and opposite charges, such as fullerene ions and dust particles. They can also help to understand numerical simulations that showed the spontaneous formation of phase-space holes in linearly stable regimes.
\end{abstract}

2000 Mathematics Subject Classification: 76X05

Keywords and Phrases: BGK modes; pair plasma 



\title{
Kinetic electrostatic structures in current-carrying pair plasmas
}

\author{
A. Luque ${ }^{1}$ H. Schamel, ${ }^{2}$ B. Eliasson, ${ }^{1}$ and P.K. Shukla ${ }^{1}$ \\ ${ }^{1}$ Institut für Theoretische Physik IV, \\ Fakultät für Physik und Astronomie, \\ Ruhr-Universität Bochum, D-44780 Bochum, Germany \\ ${ }^{2}$ Physikalisches Institut, Universität Bayreuth, D-95440 Bayreuth, Germany
}

(Dated: December 8, 2005)

\begin{abstract}
The existence and properties of phase-space structures in current-carrying pair plasmas is studied by means of the finite amplitude expressions of the pseudo-potential method. Emphasis is given to double layers, solitary structures and periodic waves. The results can be applied to electron-positron plasmas as well as to plasmas containing heavier charged particles with the same mass and opposite charges, such as fullerene ions and dust particles. They can also help to understand numerical simulations that showed the spontaneous formation of phase-space holes in linearly stable regimes.

PACS numbers: 52.35.Fp, 52.35.Sb, 52.27.Ep, 81.05.Tp
\end{abstract}

\section{INTRODUCTION}

The investigation of collective phenomena in pair plasmas, consisting of two species with the same mass is gaining interest among the plasma physics community [1]. There are several reasons for that: the first one is that electron-positron pair plasmas are present in many astrophysical contexts, such as the early universe, neutron stars and active galactic nuclei. On the other hand, recent experiments with fullerene pair plasmas $[2,3]$ have allowed the investigators to observe collective phenomena in the laboratory, something that was earlier impeded by the short annihilation times of electron-positron laboratory plasmas [4-6]. But another explanation for the outstanding interest in pair plasmas is their relatively simple theoretical description, which makes them a particularly good subject of investigations for questions in fundamental plasma theory.

This property of pair plasmas has been emphasized in earlier works that investigated the nonlinear instability [7] and saturation of current-carrying plasmas $[8,9]$. In these works, use was made of the equality of time-scales in the dynamics of both species to accelerate the onset and evolution of instability in a linearly stable regime. It was also found that, after a turbulent transient stage, the system reached a stable state in which phase-space structures were present coexisting with distribution functions one of which being characterized by a flat trapped particle distribution. Kinetic 
structures in pair plasmas were on the other hand investigated in Refs. [10] and [11], two works on which the present article relies. However, in the first, analytical one the investigations were limited to small amplitude waves and a complete symmetry in the parameters describing the distribution functions of both species whereas in the second, numerical one only solitary phase-space holes were considered. Both works did not consider current-carrying plasmas. It is thus appropiate to remove these restrictions in order to arrive at a more general theory of electrostatic trapping in pair plasmas, which is the purpose of the present article.

This paper is organized as follows. In section II we provide a theoretical background about the governing equations and the procedure to obtain finite amplitude equilibrium solutions. Sections III, IV and V are dedicated, respectively, to the study of double layers, phase-space holes and periodic solutions. Finally, the results and conclusions are summarized in section VI.

\section{THEORETICAL BACKGROUND}

We consider a drifting collisionless pair plasma with equal temperatures for both particle species. The dynamics is governed by the 1D Vlasov-Poisson system

$$
\begin{gathered}
{\left[\partial_{t}+v \partial_{x} \pm \partial_{x} \Phi(x, t) \partial_{v}\right] f_{\mp}(x, v, t)=0,} \\
\partial_{x x}^{2} \Phi(x, t)=\int d v f_{-}-\int d v f_{+} \equiv n_{-}-n_{+},
\end{gathered}
$$

where space $x$, time $t$, velocity $v$, the distribution functions $f_{ \pm}$and electric potential $\Phi$ have been normalized by the Debye length $\lambda_{D}$, the inverse plasma frequency $\omega_{p}^{-1}$, the thermal speed $V_{T}$, $n_{0} / V_{T}$ and $T / e$, respectively. Here, $\lambda_{D}=\left(n_{0} e^{2} / \epsilon_{0} T\right)^{1 / 2}, \omega_{p}=\left(n_{0} e^{2} / \epsilon_{0} m\right)^{1 / 2}$ and $V_{T}=(T / m)^{1 / 2}$, where $n_{0}$ is the unperturbed particle density of each species, $e$ is the magnitude of the electron charge, $m$ is the mass and $T$ is the temperature (in Joules) of the two species in the unperturbed state. The distribution functions of the homogeneous, unperturbed state are in the center-of-mass frame $f_{0 \mp}=(1 / \sqrt{2 \pi}) \exp \left\{-\left(v \mp v_{D} / 2\right)^{2} / 2\right\}$.

Electrostatic structures in plasmas, also called BGK-like (Bernstein-Greene-Kruskal [12]) wave structures, have been throughly investigated by means of the pseudo-potential method, first introduced in the kinetic regime in Ref. [13] and further developed in Refs. 14-16 (see also Refs. 17 and 18 for a review). In this method, the distribution functions for equilibria in the wave frame, moving at a velocity $v_{0}$ with respect to the center of mass frame, depend on the constants of motion in a 
prescribed form, namely

$$
\begin{aligned}
f_{+}(v, \Phi)= & \frac{N_{+}}{\sqrt{2 \pi}}\left\{\theta\left(\epsilon_{+}\right) \exp \left[-\frac{1}{2}\left(\sigma \sqrt{2 \epsilon_{+}}+v_{+}\right)^{2}\right]\right. \\
& \left.+\theta\left(-\epsilon_{+}\right) \exp \left(-\frac{v_{+}^{2}}{2}\right) \exp \left(-\alpha \epsilon_{+}\right)\right\}, \\
f_{-}(v, \Phi)= & \frac{N_{-}}{\sqrt{2 \pi}}\left\{\theta\left(\epsilon_{-}\right) \exp \left[-\frac{1}{2}\left(\sigma \sqrt{2 \epsilon_{-}}-v_{-}\right)^{2}\right]\right. \\
& \left.+\theta\left(-\epsilon_{-}\right) \exp \left(-\frac{v_{-}^{2}}{2}\right) \exp \left(-\beta \epsilon_{-}\right)\right\},
\end{aligned}
$$

where $\theta(z)$ represents the Heaviside step function, $\sigma=\operatorname{sg}(v)$ is the sign of the velocity, $N_{ \pm}$are normalization constants, $v_{\mp}=v_{D} / 2 \mp v_{0}$ and $\alpha$ and $\beta$ are the trapping parameters of positive and negative ions, respectively. The single particle energies, which are constants of motion, are given by $\epsilon_{\mp}:=\frac{v^{2}}{2}-\Phi_{\mp}$, where we defined $\Phi_{-}:=\Phi$ and $\Phi_{+}:=\Psi-\Phi$ respectively, where $\Psi$ is the maximum value of $\Phi$. The separatrix in the phase space of both species is then given by $\epsilon_{\mp}=0$, separating free $\left(\epsilon_{\mp}>0\right)$ from trapped $\left(\epsilon_{\mp}<0\right)$ particles.

The distribution functions given by (2) can be integrated in velocity, and yield the particle densities as functions of the electrostatic potential as $n_{+}(\Phi)=N_{+} n_{0}\left(v_{+}, \alpha, \Psi-\Phi\right), n_{-}(\Phi)=$ $N_{-} n_{0}\left(v_{-}, \beta, \Phi\right)$, where

$$
n_{0}(u, \beta, \Phi):=\exp \left(-u^{2} / 2\right)\left[F\left(u^{2} / 2, \Phi\right)+T(\beta, \Phi)\right]
$$

The definitions of the special functions $F\left(v^{2} / 2, \Phi\right)$ and $T(\beta, \Phi)$, which represent the contribution of free and trapped particles, respectively, to the density, are presented in Refs. [13, 17]. Note that $n_{0}(u, \beta, 0)=1$ for any $u, \beta$. The Poisson equation is now solved by defining the classical potential $V(\Phi)$ that satisfies $\Phi^{\prime \prime}(x)=n_{-}(\Phi)-n_{+}(\Phi)=:-\partial V(\Phi) / \partial \Phi$. Multiplying both sides by $\Phi^{\prime}(x)$ and integrating once, we have $\Phi^{\prime}(x)^{2} / 2+V(\Phi)=0$, where the classical potential is

$$
V(\Phi)=N_{+}\left[V_{0}\left(v_{+}, \alpha, \Psi\right)-V_{0}\left(v_{+}, \alpha, \Psi-\Phi\right)\right]-N_{-} V_{0}\left(v_{-}, \beta, \Phi\right)
$$

and we have defined

$$
V_{0}(u, \beta, \Phi):=\exp \left(-u^{2} / 2\right)\left[P(\beta, \Phi)-1+H\left(u^{2} / 2,0, \Phi\right)\right]
$$

We note that $V(0)=0$. The expressions for the special functions $P(\beta, \Phi)$ and $H\left(u^{2} / 2,0, \Phi\right)$ are also found in Ref. [13, 17]. To find acceptable, physical solutions two conditions have to be imposed upon $V(\Phi)$ : a) $V(\Phi) \leq 0$ if $0 \leq \Phi \leq \Psi$ and b) $V(\Psi)=0$. The second condition is usually referred to as the nonlinear dispersion relation (NDR), as it links the amplitude of the structure to its phase speed. 

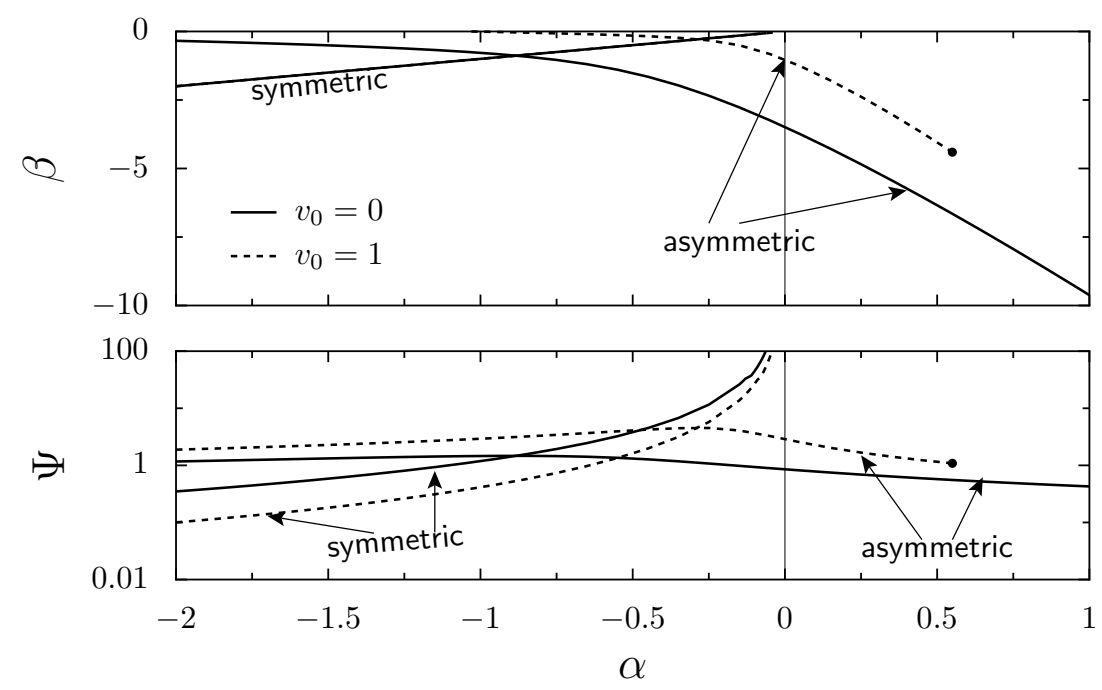

FIG. 1: Existence curves of double layers (DL) in a non-drifting pair plasma. The solid line represents standing DLs while the dashed one stands for propagating DLs with a phase speed $v_{0}=1$. Note that for both cases two branches exist, called symmetric and asymmetric branches. Note also that there are no asymmetric solutions in the propagating case if $\alpha>0.55$.

\section{DOUBLE LAYERS}

Now we make use of the expressions presented in the previous section to obtain equilibrium solutions of the Vlasov-Poisson system. First, we look for double layer (DL) solutions. Double layers are configurations of phase-space which are associated with a monotonic step-like potentials $[17,19]$.

In order to have a DL, the densities of both species must be equal as $x \rightarrow \pm \infty$, which means $\Phi \rightarrow 0$ and $\Phi \rightarrow \Psi$. Let us assume that the densities are unity at $\Phi=0$. This implies that $N_{-}=1$ and $N_{+}=1 / n_{0}\left(v_{+}, \alpha, \Psi\right)$. Under this assumption, the condition $n_{-}(\Psi)=n_{+}(\Psi)$ reduces to

$$
n_{0}\left(v_{+}, \alpha, \Psi\right) n_{0}\left(v_{-}, \beta, \Psi\right)=1
$$

This equation has to be solved simultaneously with the NDR for the unknowns $u_{0}$ and $\Psi$ in order to obtain valid solutions.

\section{A. Non-drifting plasma}

In Ref. [10], the existence of DLs in pair plasmas was discussed. They were limited, however, to the small amplitude limit and with the further assumption of complete symmetry between positive and negative particles $(\alpha=\beta)$. Here we show that, even if we drop such limitations, we can still 

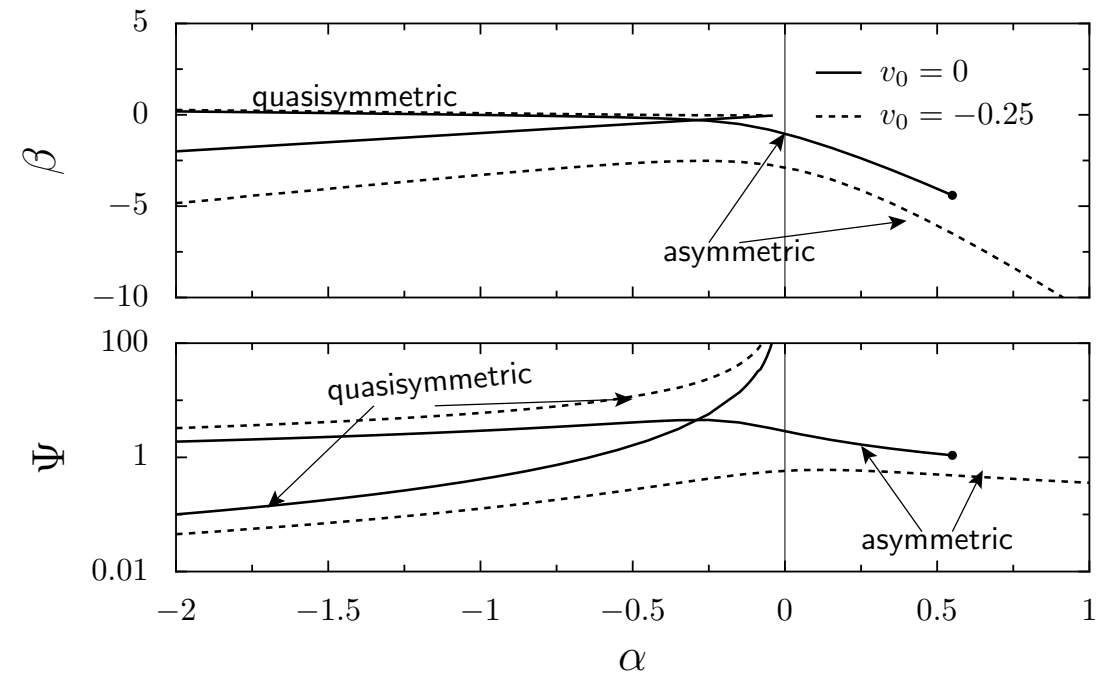

FIG. 2: Existence curves of double layers (DL) in a current-carrying pair plasma with $v_{D}=2$. The solid line represents standing DLs while the dashed one stands for propagating DL with a phase speed $v_{0}=-0.25$. Note that for both cases two branches exist, which are related to the symmetric and asymmetric branches in the case $v_{D}=0$. Note also that the curves for $v_{D}=1, v_{0}=0$ are equivalent to that of $v_{D}=0, v_{0}=1$, plotted in Fig. 1 (see text).

find DL solutions. Their existence curves are plotted in Fig. 1. Note that there are two different branches, labelled "symmetric" and "asymmetric".

The symmetric branch represents solutions for which $\alpha=\beta$. It exists only for $\alpha<0$ and admits DLs of arbitrary strength. In the limit $\Psi \ll 1(|\alpha|=|\beta| \gg 1)$ it corresponds to the solutions of Ref. [10], which, for $v_{0}=0$ satisfy $(1-\alpha)=3 \sqrt{\pi} / 4 \sqrt{\Psi}$. For finite amplitudes we observe that the relationship between the defining parameters is very well approximated by an expression of the form $|\alpha| \Psi^{\gamma}=C$, where for standing DLs $\left(v_{0}=0\right)$ we found $\gamma \approx 0.59, C \approx 1.08$. This implies that the DL becomes the stronger the more flat both trapped particle distributions are.

The asymmetric branch, on the other side, represents solutions for which the complete symmetry between the species is broken. This branch contains also solutions for which $\alpha \geq 0$. In the special case $\alpha=0$, the distribution function of positive particles is flat in the trapped range while trapped negative particles form a dip.

The most relevant physical difference between both branches is that, while solutions in the symmetric branch do not present a jump in the densities, this is not true for asymmetric solutions. 


\section{B. Current-carrying plasma}

The picture is changed when we look for double layers in a current-carrying plasma, a case which was not discussed in Ref. [10]. The parameter curves representing possible solutions for $v_{D}=2$ are shown in Fig. 2.

First of all we note that the expressions for $n_{0}(u, \beta, \Phi)$ and $V_{0}(u, \beta, \Phi)$ do only depend on $u^{2}$. Therefore we can make a complete correspondence between standing structures in a currentcarrying plasma $\left(v_{-}=v_{+}=v_{D} / 2\right)$ and propagating structures in a non-current-carrying plasma $\left(-v_{-}=v_{+}=v_{0}\right)$ just by interchanging $v_{D} / 2$ and $\pm v_{0}$. This explains that the solid lines in Fig. 2 are the same as the dashed ones in Fig. 1. Note however that, although the location of solutions in parameter space are exactly the same, both represent very different kinds of solutions.

Nevertheless, if we look for propagating structures in current-carrying plasmas, the symmetry is broken and the correspondence is no longer valid. An example of the location in parameter space of propagating DLs is given by the dashed line in Fig. 2. Note that we still have two different branches but now, as $v_{0} \neq 0$ imposes a further asymmetry, we do not have a family of solutions with $\alpha=\beta$. We can still call the two branches of solutions "asymmetric" and "quasisymmetric branch". The quasisymmetric branch exists only for $\alpha<0$ and requires increasing amplitudes as $\alpha \rightarrow 0$. Here we find also a power law of the form $|\alpha|^{\gamma} \Psi=C$ where for $v_{D}=2, v_{0}=-0.25$ we obtain $\gamma \approx 1.015, C \approx 6.11$.

\section{SOLITARY PHASE-SPACE HOLES}

The existence and properties of solitary holes in non-drifting pair plasmas was already studied in Ref. [11]. Therefore here we focus on the existence of solitary holes in current-carrying pair plasmas. As the symmetry arguments mentioned in the past section hold for holes as well as for DLs, standing holes in a current-carrying plasma correspond one-to-one to propagating holes in a plasma with $v_{D}=0$.

Without loss of generality, we will consider here holes in $f_{-}$, corresponding to bell-like electrostatic potentials, i.e. $V^{\prime}(0)=0, V^{\prime}(\Psi)>0$. We will also consider only the case of a flat trapped range in $f_{+}(\alpha=0)$. There are several reasons to give special attention to these cases: a) there are large numerical evidences that such configurations are more stable and therefore potential attractors of the dynamics. For example, in Ref. [20] the evolution of two colliding holes was simulated which resulted in a partially flat distribution function, and b) recent simulations of nonlinear insta- 


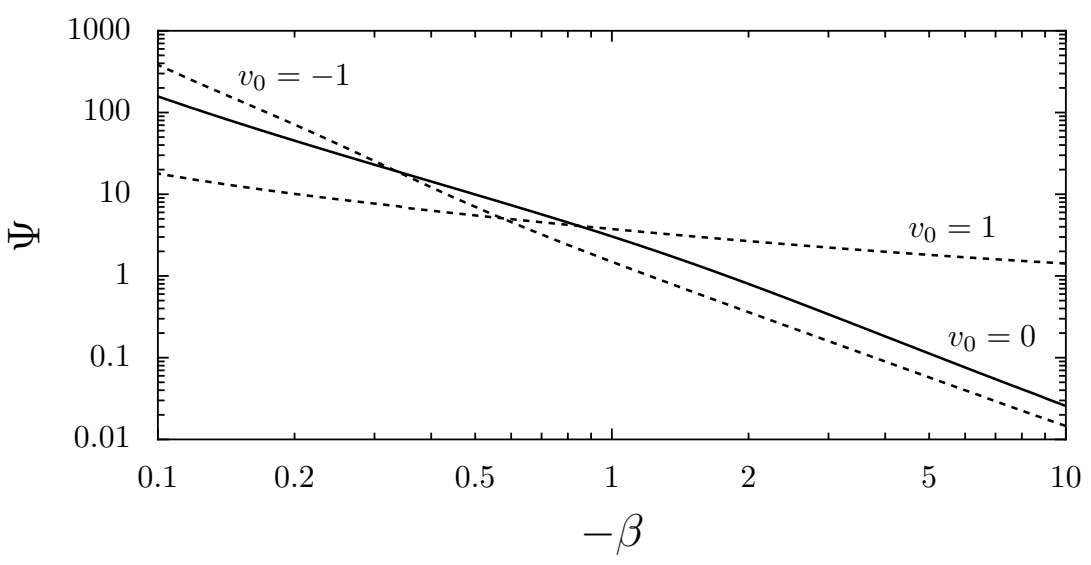

FIG. 3: The amplitude of the electrostatic potential for holes in $f_{-}$as a function of the trapping parameter $\beta$ for different phase velocities. The drift between both species was $v_{D}=2$ and a flat trapped range is assumed in $f_{+}(\alpha=0)$.

bility [9] and the subsequent turbulence in current-carrying pair plasmas show that this turbulence decays towards a stable hole equilibrium in which one of the species presents a flat distribution in the resonant range.

In Fig. 3 we present the curves in the $\beta, \Psi$ parameter space where solitary hole solutions exist in a plasma with $v_{D}=2$ for different values of the phase velocity $v_{0}$. No solutions are possible with $\beta>0$. Note that even for large amplitudes, the curves can be approximated by a power law of the form $|\beta|^{\gamma} \Psi=C$, which is actually exact for small amplitudes.

\section{PERIODIC STRUCTURES}

Periodic structures can also appear in pair plasmas. This is important because they are excited in experiments such as Ref. [2] is usually periodical and also because most numerical codes impose periodic boundary conditions in space, giving preference to periodic structures.

In Ref. [10] a dispersion relation was found for harmonic waves that smoothly joined the limits of slow acoustic modes $\omega / \sqrt{2} \approx 0.924 k$ and plasma waves $\omega / \sqrt{2} \approx 1$. To understand how the finite amplitude of the potential affects these results, we will use $\alpha=\beta=1-v_{0}^{2}$, which for small amplitudes gives raise to harmonic waves ( $B=0$ in Ref. [10]).

Figure 4 shows the dispersion relation of these waves with different values of the amplitude of the electrostatic potential. There we notice that for large amplitude waves the minimum phase velocity, corresponding to the slow acoustic mode, is considerably smaller than the one corresponding to very small amplitudes $\left(v_{0}=0.924 \sqrt{2}\right.$ when $\left.\Psi \ll 1\right)$. This slowing effect is shown in Fig. 5 , where 


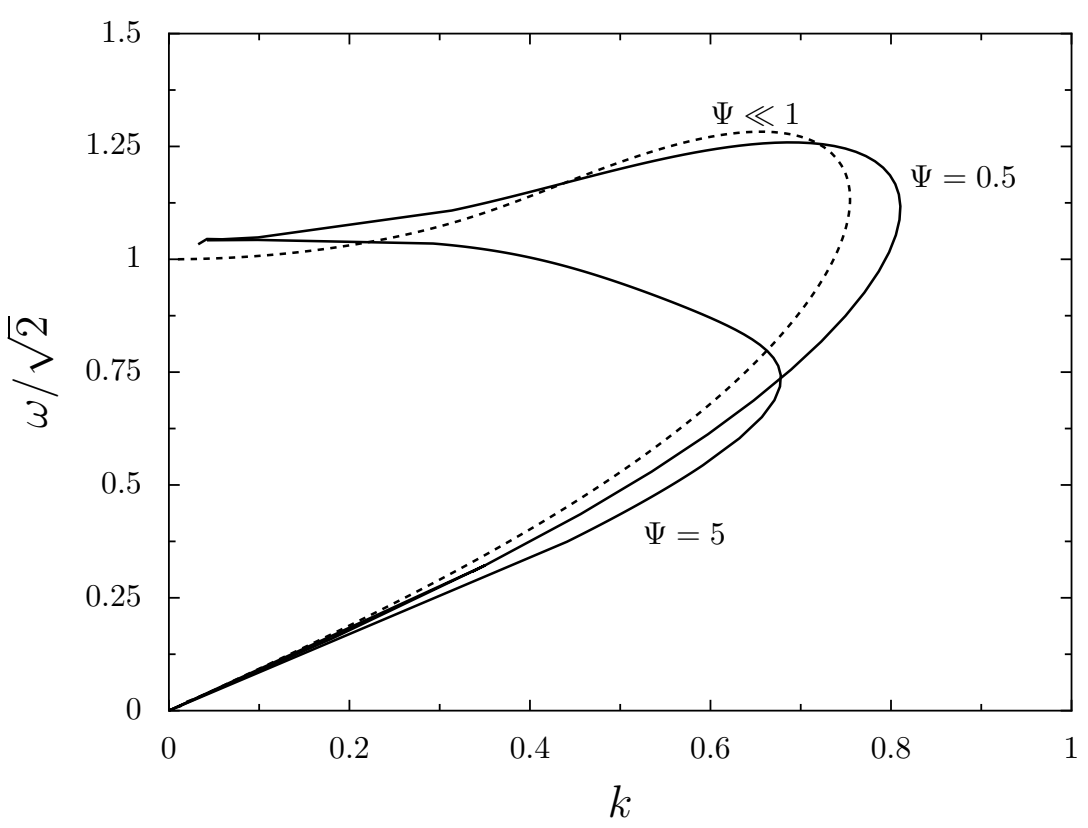

FIG. 4: Dispersion relation for waves with $\alpha=\beta=1-v_{0}^{2}$ and several amplitudes of the electrostatic potential. The dashed curve represents the limit $\Psi \ll 1$, as analytically found in Ref. [10].

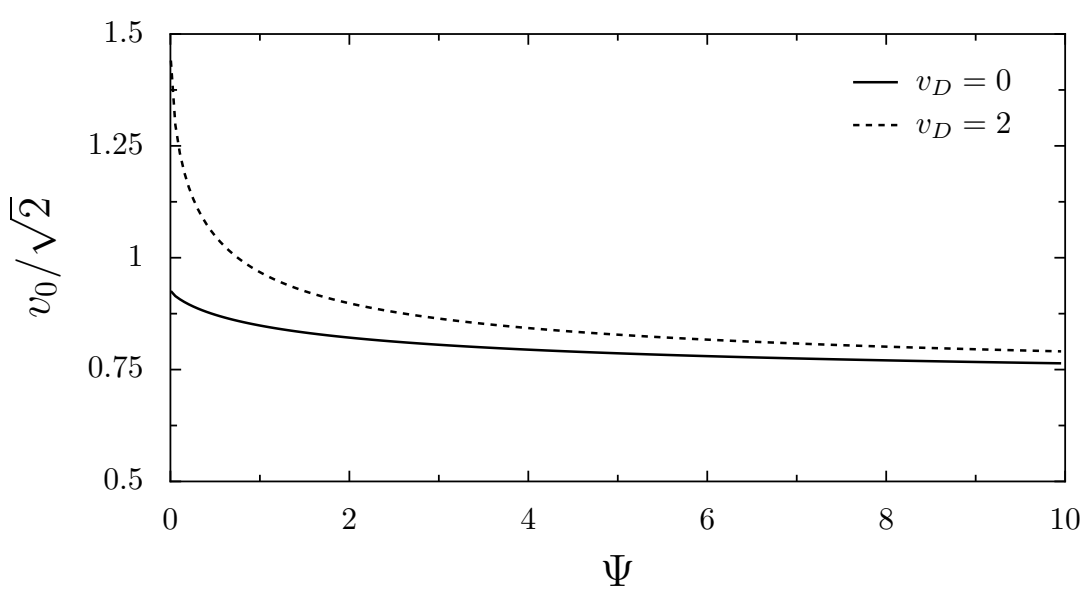

FIG. 5: Velocity of the slow acoustic branch in the limit of large wavelengths as a function of the amplitude of the electrostatic potential.

the velocity of the slow acoustic mode is plotted as a function of the amplitude of the electrostatic potential.

\section{SUMMARY AND CONCLUSIONS}

In this paper we investigated the existence of electrostatic structures in pair plasmas with a drift between the species due e.g. to an external electric field. This results extend and complement 
those of Refs.[10] and [11] in several aspects: we do not restrict ourselves to the case of perfect symmetry between the species $(\alpha=\beta)$, we do not base the results in the small amplitude limit $(\Psi \ll 1)$ and, finally we do not consider only plasmas without a drift velocity between the species $\left(v_{D}=0\right)$. By removing those limitations we have explored a wide range of possible structures. We have focused on some remarkable results that may help to interprete future experimental and numerical data.

As relevant outcome, we emphasize the existence of asymmetric double layers, that exhibit a jump in the densities, as well as the dependence between the velocity of the slow acoustic mode and the potential amplitude for periodic waves. This latter result can easily be compared with experimental data extracted from a laboratory setup like that of Ref. [2], something that we propose here and that would yield interesting results.

We would like also to underline that the results presented here are also connected with the studies about the nonlinear instability and saturation of pair plasmas, presented elsewhere [9]. The connection is twofold: a) in that reference it was shown that nonlinear stability is triggered by small amplitude phase-space holes and b) the final stable equilibrium is indeed a structured equilibrium that should be studied with the tools presented here.

\section{Acknowledgments}

This work was supported by the European Commission (Brussels) through contract No. HPRNCT-2001-00314 for carrying out the task of the RTN Network "Turbulent Boundary Layers in Geospace Plasmas", as well as by the Deutsche Forschungsgemeinschaft through the Sonderforschungsbereich 591.

[1] J. Vranjes and S. Poedts, Plasma Sources Sci. Technol. 14, 485 (2005).

[2] W. Oohara and R. Hatakeyama, Phys. Rev. Lett. 91, 205005 (2003).

[3] W. Oohara, D. Date, and R. Hatakeyama, Phys. Rev. Lett. 95, 175003 (2005).

[4] C. M. Surko, M. Leventhal, and A. Passner, Phys. Rev. Lett. 62, 901 (1989).

[5] H. Boehmer, M. Adams, and N. Rynn, Phys. Plasmas 2, 4369 (1995).

[6] E. P. Liang, S. C. Wilks, and M. Tabak, Phys. Rev. Lett. 81, 4887 (1998).

[7] R. H. Berman, D. J. Tetreault, T. H. Dupree, and T. Boutros-Ghali, Phys. Rev. Lett. 48, 1249 (1982).

[8] H. Schamel and A. Luque, Space Sci. Rev. (2005), in press.

[9] A. Luque, H. Schamel, B. Eliasson, and P. K. Shukla, Phys. Plasmas (2005), in press. 
[10] H. Schamel and A. Luque, New J. Phys. 7, 69 (2005).

[11] B. Eliasson and P. K. Shukla, Phys. Rev. E 71, 046402 (2005).

[12] I. B. Bernstein, J. M. Greene, and M. D. Kruskal, Phys. Rev. 108, 546 (1957).

[13] H. Schamel, Plasma Phys. 14, 905 (1972).

[14] H. Schamel, J. Plasma Phys. 9, 377 (1973).

[15] H. Schamel, J. Plasma Phys. 13, 139 (1975).

[16] H. Schamel, Phys. Plasmas 7, 4831 (2000).

[17] H. Schamel, Phys. Rep. 140, 161 (1986).

[18] A. Luque and H. Schamel, Phys. Rep. 415, 261 (2005).

[19] H. Schamel and S. Bujarbarua, Phys. Fluids 26, 190 (1983).

[20] B. Eliasson and P. K. Shukla, Phys. Rev. Lett. 92, 095006 (2004). 